\title{
Efficient many-party controlled teleportation of multiqubit quantum information via entanglement
}

\author{
Chui-Ping Yang, ${ }^{1,2}$ Shih-I Chu, ${ }^{2}$ and Siyuan Han ${ }^{1}$ \\ ${ }^{1}$ Department of Physics and Astronomy, University of Kansas, Lawrence, Kansas 66045, USA \\ ${ }^{2}$ Department of Chemistry, University of Kansas, and Kansas Center for Advanced Scientific Computing, \\ Lawrence, Kansas 66045, USA
}

(Received 11 December 2003; published 30 August 2004)

\begin{abstract}
We present a way to teleport multiqubit quantum information from a sender to a distant receiver via the control of many agents in a network. We show that the original state of each qubit can be restored by the receiver as long as all the agents collaborate. However, even if one agent does not cooperate, the receiver cannot fully recover the original state of each qubit. The method operates essentially through entangling quantum information during teleportation, in such a way that the required auxiliary qubit resources, local operation, and classical communication are considerably reduced for the present purpose.
\end{abstract}

DOI: 10.1103/PhysRevA.70.022329

PACS number(s): 03.67.Lx, 03.65.-w, 42.50.Dv

\section{INTRODUCTION AND MOTIVATION}

Over the past decade, scientists have made dramatic progress in the field of quantum teleportation. Theoretically, since the work of Bennett et al. [1] on teleporting a qubit of unknown information with the aid of Einstein-PodolskyRosen (EPR) correlation, quantum teleportation has been extended from discrete-variable systems to continuous-variable systems [2-5] and also from a single qubit to multiqubits [6-9]. On the other hand, recent experiments have demonstrated quantum teleportation with photon-polarized states [10], optical coherent states [11], and nuclear magnetic resonance [12].

In 1998, Karlsson and Bourennane [13] generalized the idea of Bennett $e t$ al. by the use of a three-qubit entangled Greenberger-Horne-Zeilinger (GHZ) state $|000\rangle+|111\rangle$ instead of an EPR pair. In their work, they showed that an arbitrary unknown state of a qubit could be teleported to either one of two receivers. But only one of the two (either one) can fully reconstruct the qubit state conditioned on the measurement outcome of the other. Since that work, Hillery et al. [14] first proposed the idea of quantum secret sharing-i.e., splitting a message into several parts so that no subset of parts is sufficient to read the message, but the entire set is. In their work, they showed how a qubit of information can be secretly shared by two agents via a three-qubit GHZ state and also generalized this procedure to split a qubit of information among more than two agents through a fourqubit GHZ state. More recently, a number of works on quantum secret sharing have also been proposed [15-20].

In this paper, we restrict ourselves to an issue-i.e., teleporting multiqubit information from a sender to a distant receiver via the control of many agents in a network. We wish that the receiver can successfully get access to the original state of each qubit, as long as all the agents collaborate through local operation and classical communication. However, even if one agent does not cooperate, the original state of each qubit cannot fully be recovered by the receiver. The topic here might be of particular interest, since controlled teleportation is useful in networked quantum information processing and cryptographic conferencing [21,22].

One possible approach is to use the method described in Refs. [13,14] directly for the present task. To explore the feasibility of this approach, let us first give a brief review of the procedure introduced in $[13,14]$. To teleport an arbitrary unknown state $|\psi\rangle_{A}=\alpha|0\rangle_{A}+\beta|1\rangle_{A}$ of one qubit $A$ from Alice to a receiver Bob via the control of $n$ agents in a network, the method in $[13,14]$ requires a $(n+2)$-qubit GHZ state $|\mathrm{GHZ}\rangle$ $=|0\rangle_{a}|0\rangle_{b}|0\rangle^{\otimes n}+|1\rangle_{a}|1\rangle_{b}|1\rangle^{\otimes n}$ shared by Alice, Bob, and the $n$ agents. Here, GHZ qubits $a$ and $b$ belong to Alice and Bob, respectively, while the other $n$ GHZ qubits belong to the $n$ agents. The initial state $|\psi\rangle_{A} \otimes|\mathrm{GHZ}\rangle$ for the whole system can be rewritten as

$$
\begin{aligned}
& \left|\Phi^{+}\right\rangle_{A a}\left(\alpha|0\rangle_{b}|0\rangle^{\otimes n}+\beta|1\rangle_{b}|1\rangle^{\otimes n}\right)+\left|\Phi^{-}\right\rangle_{A a}\left(\alpha|0\rangle_{b}|0\rangle^{\otimes n}\right. \\
& \left.\quad-\beta|1\rangle_{b}|1\rangle^{\otimes n}\right)+\left|\psi^{+}\right\rangle_{A a}\left(\alpha|1\rangle_{b}|1\rangle^{\otimes n}+\beta|0\rangle_{b}|0\rangle^{\otimes n}\right) \\
& \quad+\left|\psi^{-}\right\rangle_{A a}\left(\alpha|1\rangle_{b}|1\rangle^{\otimes n}-\beta|0\rangle_{b}|0\rangle^{\otimes n}\right)
\end{aligned}
$$

where $\left|\Phi^{ \pm}\right\rangle_{A a}=|00\rangle_{A a} \pm|11\rangle_{A a}$ and $\left|\psi^{ \pm}\right\rangle_{A a}=|01\rangle_{A a} \pm|10\rangle_{A a}$ are Bell states for the two qubits $A$ and $a$. Based on Eq. (1), the following can be shown.

(i) For every outcome of Alice's Bell-state measurement on her qubits $A$ and $a$, Bob can restore the original state $\alpha|0\rangle_{A}+\beta|1\rangle_{A}$ of the message qubit $A$ through his qubit $b$, provided that each agent cooperates with him. That is, each agent performs a Hadamard transformation $|0\rangle \rightarrow|0\rangle+|1\rangle$ and $|1\rangle \rightarrow|0\rangle-|1\rangle$ on his or her GHZ qubit, then makes a measurement on his or her GHZ qubit in a single-qubit computational basis $|0\rangle$ and $|1\rangle$, a measurement basis in which all single-qubit measurements discussed throughout this paper will be performed, and finally sends his or her measurement result (one-bit classical message) to Bob.

(ii) On the other hand, note that after Alice's Bell-state measurement, the $n+1$ parties (the $n$ agents plus Bob) are left sharing a $(n+1)$-qubit state of the form $\alpha|0\rangle_{b}|0\rangle^{\otimes n} \pm \beta|1\rangle_{b}|1\rangle^{\otimes n}$ or $\alpha|1\rangle_{b}|1\rangle^{\otimes n} \pm \beta|0\rangle_{b}|0\rangle^{\otimes n}$ (depending on Alice's Bell-state measurement outcome). Thus, even if one agent does not cooperate with Bob, the resulting density operator for the qubit $b$ belonging to Bob would be $\rho_{b}$ 
$=|\alpha|^{2}|0\rangle_{b}\left\langle\left. 0|+| \beta\right|^{2} \mid 1\right\rangle_{b}\langle 1|$ or $|\alpha|^{2}|1\rangle_{b}\left\langle\left. 1|+| \beta\right|^{2} \mid 0\right\rangle_{b}\langle 0|$, which implies that Bob has amplitude information about Alice's message qubit $A$ but knows nothing about its phase. Therefore, Bob cannot fully gain the original information of Alice's qubit $A$ even if one agent does not collaborate with him.

Note that the above argument is for the controlled teleportation of one-qubit information. The procedure to extend the method in $[13,14]$ to teleport $m$-qubit information from a sender to a distant receiver via the control of $n$ agents is as follows. First, the sender needs to prepare $m$ copies of a $(n$ +2)-qubit GHZ state and then send each agent one GHZ qubit for every $(n+2)$-qubit GHZ state; i.e., a total of $m \mathrm{GHZ}$ qubits are needed to be distributed to each agent. Second, each agent needs to perform a Hadamard transformation and then measurement on each of his or her $m$ GHZ qubits; i.e., a total of $m$ single-qubit Hadamard transformations and $m$ single-qubit measurements are required for each agent. Last, each agent needs to send the receiver all of his or her measurement results (i.e., $m$-bit classical message), in order for the receiver to restore the original $m$-qubit information. Hence, the method in $[13,14]$ requires considerably large auxiliary qubit resources and local operation, as well as classical communication, especially when the number of "teleported"-message qubits is significantly large.

In the following, we describe a way to implement the present task. The method works actually by entangling quantum information during teleportation. As shown below, regardless of the amount of information to be teleported, the proposed approach only requires that (i) the sender assign one qubit to each agent, (ii) each agent perform one singlequbit Hadamard transformation and one single-qubit measurement on his or her qubit, and (iii) each agent send one-bit classical message to the receiver. Therefore, compared with the method in $[13,14]$, the present scheme is much simpler and economical, because the required auxiliary qubit resources, the number of local operations, and the quantity of classical communication are greatly reduced.

The paper is organized as follows. In Sec. II, we present a way to teleport multiqubit information to a distant receiver via the control of one agent. We then make a comparison between our method and the method in $[13,14]$. In Sec. III, we discuss how to decompose multiqubit GHZ states and then generalize our method to a multiagent controlled teleportation. In Sec. IV, we further apply the method to teleporting a multiple qubit-string message to many distant receivers via the control of many agents in a network. A brief discussion and the concluding summary are given in Sec. V.

\section{TELEPORTATION OF MULTIQUBIT INFORMATION TO A DISTANT RECEIVER VIA THE CONTROL OF ONE AGENT}

Suppose that Alice holds a string of message qubits labeled by $1,2, \ldots, m$, which is initially in the state $\prod_{i=1}^{m}\left(\alpha_{i}|0\rangle_{i}+\beta_{i}|1\rangle_{i}\right)$. She wishes to send $m$-qubit information to a distant receiver (Bob) via the control of one agent (Carol), such that Bob can get the complete information carried by each message qubit only if Carol collaborates. This can be done by the following procedure
First, Alice prepares the following EPR entangled state through local logic gates:

$$
\begin{aligned}
& \prod_{i=1}^{m}\left(|00\rangle_{i^{\prime} i^{\prime \prime}}+|11\rangle_{i^{\prime} i^{\prime \prime}}\right) \otimes\left(|00\rangle_{a c}+|11\rangle_{a c}\right)+\prod_{i=1}^{m}\left(|00\rangle_{i^{\prime} i^{\prime \prime}}\right. \\
& \left.-|11\rangle_{i^{\prime} i^{\prime \prime}}\right) \otimes\left(|00\rangle_{a c}-|11\rangle_{a c}\right),
\end{aligned}
$$

and then sends EPR qubit $c$ to Carol and $m$ EPR qubits $\left(1^{\prime \prime}, 2^{\prime \prime}, \ldots, m^{\prime \prime}\right)$ to Bob, while keeping the other $m+1$ EPR qubits $\left(1^{\prime}, 2^{\prime}, \ldots, m^{\prime}\right)$ and $a$ to herself. The state of the whole system is given by

$$
\begin{aligned}
& \prod_{i=1}^{m}\left[\left(\alpha_{i}|0\rangle_{i}+\beta_{i}|1\rangle_{i}\right)\left(|00\rangle_{i^{\prime} i^{\prime \prime}}+|11\rangle_{i^{\prime} i^{\prime \prime}}\right)\right] \otimes\left(|00\rangle_{a c}+|11\rangle_{a c}\right) \\
& +\prod_{i=1}^{m}\left[\left(\alpha_{i}|0\rangle_{i}+\beta_{i}|1\rangle_{i}\right)\left(|00\rangle_{i^{\prime} i^{\prime \prime}}-|11\rangle_{i^{\prime} i^{\prime \prime}}\right)\right] \otimes\left(|00\rangle_{a c}-|11\rangle_{a c}\right),
\end{aligned}
$$

which can be written as

$$
\begin{aligned}
& \prod_{i=1}^{m}\left[\left|\phi_{i i^{\prime}}^{+}\right\rangle\left(\alpha_{i}|0\rangle_{i^{\prime \prime}}+\beta_{i}|1\rangle_{i^{\prime \prime}}\right)+\left|\phi_{i i^{\prime}}^{-}\right\rangle\left(\alpha_{i}|0\rangle_{i^{\prime \prime}}-\beta_{i}|1\rangle_{i^{\prime \prime}}\right)+\left|\psi_{i i^{\prime}}^{+}\right\rangle\right. \\
& \left.\times\left(\alpha_{i}|1\rangle_{i^{\prime \prime}}+\beta_{i}|0\rangle_{i^{\prime \prime}}\right)+\left|\psi_{i i^{\prime}}^{-}\right\rangle\left(\alpha_{i}|1\rangle_{i^{\prime \prime}}-\beta_{i}|0\rangle_{i^{\prime \prime}}\right)\right] \otimes\left(|00\rangle_{a c}\right. \\
& \left.+|11\rangle_{a c}\right)+\prod_{i=1}^{m}\left[\left|\phi_{i i^{\prime}}^{+}\right\rangle\left(\alpha_{i}|0\rangle_{i^{\prime \prime}}-\beta_{i}|1\rangle_{i^{\prime \prime}}\right)+\left|\phi_{i i^{\prime}}^{-}\right\rangle\left(\alpha_{i}|0\rangle_{i^{\prime \prime}}\right.\right. \\
& \left.+\beta_{i}|1\rangle_{i^{\prime \prime}}\right)+\left|\psi_{i i^{\prime}}^{+}\right\rangle\left(-\alpha_{i}|1\rangle_{i^{\prime \prime}}+\beta_{i}|0\rangle_{i^{\prime \prime}}\right)+\left|\psi_{i i^{\prime}}^{-}\right\rangle\left(-\alpha_{i}|1\rangle_{i^{\prime \prime}}\right. \\
& \left.\left.-\beta_{i}|0\rangle_{i^{\prime \prime}}\right)\right] \otimes\left(|00\rangle_{a c}-|11\rangle_{a c}\right) .
\end{aligned}
$$

Here and below, the subscripts $i i^{\prime}=11^{\prime}, 22^{\prime}, 33^{\prime} \ldots ; i^{\prime} i^{\prime \prime}$ $=1^{\prime} 1^{\prime \prime}, 2^{\prime} 2^{\prime \prime}, 3^{\prime} 3^{\prime \prime} \ldots$; and $i^{\prime \prime}=1^{\prime \prime}, 2^{\prime \prime}, 3^{\prime \prime} \ldots$; for $i=1,2,3 \ldots$. In addition, normalized factors throughout this paper are omitted for simplicity. The states $\left|\phi_{i i^{\prime}}^{+}\right\rangle,\left|\phi_{i i^{\prime}}^{-}\right\rangle,\left|\psi_{i i^{\prime}}^{+}\right\rangle$, and $\left|\psi_{i i^{\prime}}^{-}\right\rangle$ involved in Eq. (4) are the four Bell states for the qubit pair $\left(i, i^{\prime}\right)$, which form a set of the complete orthogonal basis in the 4D Hilbert space of the two qubits $i$ and $i^{\prime}$, and take the following expressions:

$$
\left|\phi_{i i^{\prime}}^{ \pm}\right\rangle=|00\rangle_{i i^{\prime}} \pm|11\rangle_{i i^{\prime}},\left|\psi_{i i^{\prime}}^{ \pm}\right\rangle=|01\rangle_{i i^{\prime}} \pm|10\rangle_{i i^{\prime}},
$$

respectively.

Second, Alice performs a series of two-qubit Bell-state measurements, respectively, on qubit pairs $\left(1,1^{\prime}\right),\left(2,2^{\prime}\right), \ldots$, $\left(m, m^{\prime}\right)$. After that, one has

$$
|\psi\rangle\left(|00\rangle_{a c}+|11\rangle_{a c}\right)+\left|\psi^{\prime}\right\rangle\left(|00\rangle_{a c}-|11\rangle_{a c}\right),
$$

with

$$
|\psi\rangle=\prod_{i=1}^{m}|\psi\rangle_{i^{\prime \prime}},\left|\psi^{\prime}\right\rangle=\prod_{i=1}^{m}\left|\psi^{\prime}\right\rangle_{i^{\prime \prime}}
$$

where $|\psi\rangle$ and $\left|\psi^{\prime}\right\rangle$ are the states for the $m$ qubits $\left(1^{\prime \prime}, 2^{\prime \prime}, \ldots, m^{\prime \prime}\right)$ belonging to Bob, while $|\psi\rangle_{i^{\prime \prime}}$ and $|\psi\rangle_{i^{\prime \prime}}^{\prime}$ are the states of Bob's qubit $i^{\prime \prime}$. From Eq.(4), one can see that the states $|\psi\rangle_{i^{\prime \prime}}$ and $|\psi\rangle_{i^{\prime \prime}}^{\prime}$ depend on the outcome of Alice's Bell- 
state measurement on the qubit pair $\left(i, i^{\prime}\right)$ and are given by

$$
\begin{gathered}
|\psi\rangle_{i^{\prime \prime}}= \begin{cases}\alpha_{i}|0\rangle_{i^{\prime \prime}}+\beta_{i}|1\rangle_{i^{\prime \prime}} & \text { for } P_{i i^{\prime}}=\left|\phi_{i i^{\prime}}^{+}\right\rangle\left\langle\phi_{i i^{\prime}}^{+}\right|, \\
\alpha_{i}|0\rangle_{i^{\prime \prime}}-\beta_{i}|1\rangle_{i^{\prime \prime}} & \text { for } P_{i i^{\prime}}=\left|\phi_{i i^{\prime}}^{-}\right\rangle\left\langle\phi_{i i^{\prime}}^{-}\right|, \\
\alpha_{i}|1\rangle_{i^{\prime \prime}}+\beta_{i}|0\rangle_{i^{\prime \prime}} & \text { for } P_{i i^{\prime}}=\left|\psi_{i i^{\prime}}^{+}\right\rangle\left\langle\psi_{i i^{\prime}}^{+}\right|, \\
\alpha_{i}|1\rangle_{i^{\prime \prime}}-\beta_{i}|0\rangle_{i^{\prime \prime}} & \text { for } P_{i i^{\prime}}=\left|\psi_{i i^{\prime}}^{-}\right\rangle\left\langle\psi_{i i^{\prime}}^{-}\right|,\end{cases} \\
\left|\psi^{\prime}\right\rangle_{i^{\prime \prime}}= \begin{cases}\alpha_{i}|0\rangle_{i^{\prime \prime}}-\beta_{i}|1\rangle_{i^{\prime \prime}} & \text { for } P_{i i^{\prime}}=\left|\phi_{i i^{\prime}}^{+}\right\rangle\left\langle\phi_{i i^{\prime}}^{+}\right|, \\
\alpha_{i}|0\rangle_{i^{\prime \prime}}+\beta_{i}|1\rangle_{i^{\prime \prime}} & \text { for } P_{i i^{\prime}}=\left|\phi_{i i^{\prime}}^{-}\right\rangle\left\langle\phi_{i i^{\prime}}^{-}\right|, \\
-\alpha_{i}|1\rangle_{i^{\prime \prime}}+\beta_{i}|0\rangle_{i^{\prime \prime}} & \text { for } P_{i i^{\prime}}=\left|\psi_{i i^{\prime}}^{+}\right\rangle\left\langle\psi_{i i^{\prime}}^{+}\right|, \\
-\alpha_{i}|1\rangle_{i^{\prime \prime}}-\beta_{i}|0\rangle_{i^{\prime \prime}} & \text { for } P_{i i^{\prime}}=\left|\psi_{i i^{\prime}}^{-}\right\rangle\left\langle\psi_{i i^{\prime}}^{-}\right|,\end{cases}
\end{gathered}
$$

where $P_{i i^{\prime}}$ is a projector onto the Bell state $\left|\phi_{i i^{\prime}}^{+}\right\rangle,\left|\phi_{i i^{\prime}}^{-}\right\rangle,\left|\psi_{i i^{\prime}}^{+}\right\rangle$, or $\left|\psi_{i i^{\prime}}^{-}\right\rangle$of the two qubits in the pair $\left(i, i^{\prime}\right)$.

The results (8) and (9) show that according to the outcome of Alice's Bell-state measurement on the pair $\left(i, i^{\prime}\right)$, Bob can always recover the original state $\alpha_{i}|0\rangle_{i}+\beta_{i}|1\rangle_{i}$ of the message qubit $i$ from the state $|\psi\rangle_{i^{\prime \prime}}$ or $\left|\psi^{\prime}\right\rangle_{i^{\prime \prime}}$ of his qubit $i^{\prime \prime}$ by performing a single-qubit operation on the qubit $i^{\prime \prime}$. For instance, Bob obtains $U|\psi\rangle_{i^{\prime \prime}} \rightarrow \alpha_{i}|0\rangle_{i^{\prime \prime}}+\beta_{i}|1\rangle_{i^{\prime \prime}}$ and $U^{\prime}\left|\psi^{\prime}\right\rangle_{i^{\prime \prime}}$ $\rightarrow \alpha_{i}|0\rangle_{i^{\prime \prime}}+\beta_{i}|1\rangle_{i^{\prime \prime}}$, where $U$ and $U^{\prime}$ are unitary operators and $U, U^{\prime}=I, \sigma_{z} ; \sigma_{z}, I ; \sigma_{x}, \sigma_{y}$; or $\sigma_{y}, \sigma_{x}$ for the case of Alice measuring the pair $\left(i, i^{\prime}\right)$ in the Bell state $\left|\phi_{i i^{\prime}}^{+}\right\rangle,\left|\phi_{i i^{\prime}}^{-}\right\rangle,\left|\phi_{i i^{\prime}}^{+}\right\rangle$, or $\left|\psi_{i i^{\prime}}^{-}\right\rangle$. Here, $\sigma_{x}, \sigma_{y}$, and $\sigma_{z}$ are Pauli operators which correspond to the rotations by $\pi$ rad about the $x, y$, and $z$ axes, respectively, and $I$ is an identity operator.

Third, Alice and Carol perform a Hadamard transformation on their respective qubits $a$ and $c$. As a result, the state $|00\rangle_{a c}-|11\rangle_{a c}$ goes to $|01\rangle_{a c}+|10\rangle_{a c}$ while the state $|00\rangle_{a c}$ $+|11\rangle_{a c}$ remains unchanged. Thus, the state (6) will, after Alice's and Carol's Hadamard transformations, change into

$$
|\psi\rangle\left(|00\rangle_{a c}+|11\rangle_{a c}\right)+\left|\psi^{\prime}\right\rangle\left(|01\rangle_{a c}+|10\rangle_{a c}\right) .
$$

Fourth, Alice and Carol make a measurement on their respective qubits $a$ and $c$, and then each sends the measurement result (one-bit classical message) to Bob. One can see from Eq. (10) that if Bob knows that Alice and Carol both measured their qubits in the state $|0\rangle$ or $|1\rangle$, he can predict that his $m$ qubits $\left(1^{\prime \prime}, 2^{\prime \prime}, \ldots, m^{\prime \prime}\right)$ must be in the state $|\psi\rangle$. On the other hand, expression (10) shows that if Bob knows that Alice measured her qubit in the state $|0\rangle(|1\rangle)$, while Carol measured his qubit in the state $|1\rangle(|0\rangle)$, he knows that his $m$ qubits must be in the state $\left|\psi^{\prime}\right\rangle$. Therefore, according to the measurement results from Alice and Carol, Bob can predict whether his $m$ qubits are in $|\psi\rangle$ or $\left|\psi^{\prime}\right\rangle$.

Last, note that the state $|\psi\rangle\left(\left|\psi^{\prime}\right\rangle\right)$, described in Eq. (7), is a product of individual-qubit states $|\psi\rangle_{1^{\prime \prime}},|\psi\rangle_{2^{\prime \prime}}, \ldots|\psi\rangle_{m^{\prime \prime}}$ $\left(\left|\psi^{\prime}\right\rangle_{1^{\prime \prime}},\left|\psi^{\prime}\right\rangle_{2^{\prime \prime}}, \ldots,\left|\psi^{\prime}\right\rangle_{m^{\prime \prime}}\right)$ for the qubits $\left(\left(1^{\prime \prime}, 2^{\prime \prime}, \ldots, m^{\prime \prime}\right)\right.$. And as addressed above, Bob can recover the original state (i.e., $\alpha_{i}|0\rangle_{i}+\beta_{i}|1\rangle_{i}$ of message qubit $i$ ) from the state $|\psi\rangle_{i^{\prime \prime}}$ or $\left|\psi^{\prime}\right\rangle_{i^{\prime \prime}}$ of his qubit $i^{\prime \prime}$, based on the outcome of Alice's Bellstate measurement on the qubit pair $\left(i, i^{\prime}\right)$ and via a singlequbit unitary transformation $U$ or $U^{\prime}$ on the qubit $i^{\prime \prime}$. Hence, Bob can always reconstruct the original state of $m$ message qubits $(1,2, \ldots, m)$ from the state $|\psi\rangle$ or $\left|\psi^{\prime}\right\rangle$ of his $m$ qubits $\left(1^{\prime \prime}, 2^{\prime \prime}, \ldots, m^{\prime \prime}\right)$, according to the outcome of Alice's Bellstate measurements on the qubit pairs $\left(1,1^{\prime}\right),\left(2,2^{\prime}\right), \ldots$, $\left(m, m^{\prime}\right)$ and through his local single-qubit operations.

In what follows, our purpose is to show that when Carol does not collaborate, it is impossible for Bob to gain the full quantum message. Examining the state (6), we see that when only Alice performs a Hadamard transformation on her qubit $a$, the state (6) will change into

$$
\begin{aligned}
& {\left[\left(|\psi\rangle+\left|\psi^{\prime}\right\rangle\right)|0\rangle_{c}+\left(|\psi\rangle-\left|\psi^{\prime}\right\rangle\right)|1\rangle_{c}\right]|0\rangle_{a}+\left[\left(|\psi\rangle+\left|\psi^{\prime}\right\rangle\right)|0\rangle_{c}\right.} \\
& \left.\quad-\left(|\psi\rangle-\left|\psi^{\prime}\right\rangle\right)|1\rangle_{c}\right]|1\rangle_{a},
\end{aligned}
$$

which implies that whether Alice measures her qubit $a$ in the state $|0\rangle$ or $|1\rangle$, the density operator of the $m$ qubits $\left(1^{\prime \prime}, 2^{\prime \prime}, \ldots, m^{\prime \prime}\right)$ belonging to Bob will, after tracing over Carol's qubit $c$, be given by

$$
\rho=\left(|\psi\rangle+\left|\psi^{\prime}\right\rangle\right)\left(\left\langle\psi\left|+\left\langle\psi^{\prime}\right|\right)+\left(|\psi\rangle-\left|\psi^{\prime}\right\rangle\right)\left(\langle\psi|-\left\langle\psi^{\prime}\right|\right) .\right.\right.
$$

The result (12) shows that the $m$ qubits $\left(1^{\prime \prime}, 2^{\prime \prime}, \ldots, m^{\prime \prime}\right)$ are in a mixed state, in which they are in a superposition state $|\psi\rangle+\left|\psi^{\prime}\right\rangle$ with a probability $\left.p_{1}=\| \psi\right\rangle+\left.\left|\psi^{\prime}\right\rangle\right|^{2} / 2(\langle\psi \mid \psi\rangle$ $\left.+\left\langle\psi^{\prime} \mid \psi^{\prime}\right\rangle\right)$ while being in the other superposition state $|\psi\rangle$ $-\left|\psi^{\prime}\right\rangle$ with a probability $\left.p_{2}=\| \psi\right\rangle-\left.\left|\psi^{\prime}\right\rangle\right|^{2} / 2\left(\langle\psi \mid \psi\rangle+\left\langle\psi^{\prime} \mid \psi^{\prime}\right\rangle\right)$.

Based on Eqs. (7)-(9), one can express the states $|\psi\rangle$ $+\left|\psi^{\prime}\right\rangle$ and $|\psi\rangle-\left|\psi^{\prime}\right\rangle$ involved in Eq. (12) as follows:

$$
\begin{aligned}
|\psi\rangle \pm\left|\psi^{\prime}\right\rangle= & |\widetilde{\psi}\rangle\left(\alpha_{t}|0\rangle_{t^{\prime \prime}}+\beta_{t}|1\rangle_{t^{\prime \prime}}\right) \pm\left|\widetilde{\psi^{\prime}}\right\rangle\left(\alpha_{t}|0\rangle_{t^{\prime \prime}}\right. \\
& \left.-\beta_{t}|1\rangle_{t^{\prime \prime}}\right) \text { for } P_{t t^{\prime}}=\left|\phi_{t t^{\prime}}^{+}\right\rangle\left\langle\phi_{t t^{\prime}}^{+}\right|, \\
|\psi\rangle \pm\left|\psi^{\prime}\right\rangle= & \left.\left|\widetilde{\psi\rangle}\left(\alpha_{t}|0\rangle_{t^{\prime \prime}}-\beta_{t}|1\rangle_{t^{\prime \prime}}\right) \pm\right| \widetilde{\psi^{\prime}}\right\rangle\left(\alpha_{t}|0\rangle_{t^{\prime \prime}}\right. \\
& \left.+\beta_{t}|1\rangle_{t^{\prime \prime}}\right) \text { for } P_{t t^{\prime}}=\left|\phi_{t t^{\prime}}^{-}\right\rangle\left\langle\phi_{t t^{\prime}}^{-}\right|, \\
|\psi\rangle \pm\left|\psi^{\prime}\right\rangle= & |\widetilde{\psi}\rangle\left(\alpha_{t}|1\rangle_{t^{\prime \prime}}+\beta_{t}|0\rangle_{t^{\prime \prime}}\right) \pm\left|\widetilde{\psi^{\prime}}\right\rangle\left(-\alpha_{t}|1\rangle_{t^{\prime \prime}}\right. \\
& \left.+\beta_{t}|0\rangle_{t^{\prime \prime}}\right) \text { for } P_{t t^{\prime}}=\left|\psi_{t t^{\prime}}^{+}\right\rangle\left\langle\psi_{t t^{\prime}}^{+}\right|, \\
|\psi\rangle \pm\left|\psi^{\prime}\right\rangle= & |\widetilde{\psi}\rangle\left(\alpha_{t}|1\rangle_{t^{\prime \prime}}-\beta_{t}|0\rangle_{t^{\prime \prime}}\right) \pm\left|\widetilde{\psi^{\prime}}\right\rangle\left(-\alpha_{t}|1\rangle_{t^{\prime \prime}}\right. \\
& \left.-\beta_{t}|0\rangle_{t^{\prime \prime}}\right) \text { for } P_{t t^{\prime}}=\left|\psi_{t t^{\prime}}^{-}\right\rangle\left\langle\left\langle\psi_{t t^{\prime}}^{-}\right|,\right.
\end{aligned}
$$

where the subscript $t^{\prime \prime}$ represents any one of the $m$ qubits $\left(1^{\prime \prime}, 2^{\prime \prime}, \ldots, m^{\prime \prime}\right)$ belonging to Bob, and the subscripts $t$ and $t^{\prime}$ denote Alice's message qubit and her EPR qubit (corresponding to Bob's qubit $t^{\prime \prime}$ ), respectively. In Eq. (13), we further note that $|\widetilde{\psi}\rangle=\Pi_{k}|\psi\rangle_{k^{\prime \prime}}$ and $\left|\widetilde{\psi^{\prime}}\right\rangle=\Pi_{k}\left|\psi^{\prime}\right\rangle_{k^{\prime \prime}}(k \neq t)$ are the states of the remaining $m-1$ qubits belonging to Bob (after excluding the qubit $t^{\prime \prime}$ ). Here, $|\psi\rangle_{k^{\prime \prime}}$ and $\left|\psi^{\prime}\right\rangle_{k^{\prime \prime}}$ are the states of qubit $k^{\prime \prime}\left(k^{\prime \prime} \neq t^{\prime \prime}\right)$, which depend on the outcome of Alice's Bell-state measurement on the pair $\left(k, k^{\prime}\right)$ and take the form of Eqs. (8) and (9), respectively. From Eqs. (12) and (13), it is easily shown that for each outcome $\left(\left|\phi_{t t^{\prime}}^{+}\right\rangle,\left|\phi_{t t^{-}}^{-}\right\rangle\right.$, $\left|\psi_{t t^{\prime}}^{+}\right\rangle$, or $\left.\left|\psi_{t t^{\prime}}^{-}\right\rangle\right)$of Alice's Bell-state measurement on the pair $\left(t, t^{\prime}\right)$, the density operator for the remaining $m-1$ qubits 
belonging to Bob is, after tracing over the qubit $t^{\prime \prime}$, given by

$$
\begin{aligned}
\widetilde{\rho}= & \operatorname{tr}_{t^{\prime \prime}}(\rho)=\left(|\widetilde{\psi}\rangle+\left|\widetilde{\psi^{\prime}}\right\rangle\right)\left(\left\langle\widetilde{\psi}\left|+\left\langle\widetilde{\psi^{\prime}}\right|\right)\left|\alpha_{t}\right|^{2}+\left(|\widetilde{\psi}\rangle+\left|\widetilde{\psi^{\prime}}\right\rangle\right)(\langle\widetilde{\psi}|\right.\right. \\
& \left.+\left\langle\widetilde{\psi^{\prime}}\right|\right)\left|\beta_{t}\right|^{2}+\left(|\widetilde{\psi}\rangle-\left|\widetilde{\psi^{\prime}}\right\rangle\right)\left(\left\langle\widetilde{\psi}\left|-\left\langle\widetilde{\psi^{\prime}}\right|\right)\left|\alpha_{t}\right|^{2}+\left(|\widetilde{\psi}\rangle-\left|\widetilde{\psi^{\prime}}\right\rangle\right)\right.\right. \\
& \times\left(\left\langle\widetilde{\psi}\left|-\left\langle\widetilde{\psi^{\prime}}\right|\right)\left|\beta_{t}\right|^{2}=\left(|\widetilde{\psi}\rangle+\left|\widetilde{\psi^{\prime}}\right\rangle\right)\left(\left\langle\widetilde{\psi}\left|+\left\langle\widetilde{\psi^{\prime}}\right|\right)+\left(|\widetilde{\psi}\rangle-\left|\widetilde{\psi^{\prime}}\right\rangle\right)\right.\right.\right.\right. \\
& \times\left(\langle\widetilde{\psi}|-\left\langle\widetilde{\psi^{\prime}}\right|\right),
\end{aligned}
$$

where we have used $\left|\alpha_{t}\right|^{2}+\left|\beta_{t}\right|^{2}=1$. Equation (14) implies that the density operator, for the remaining $m-1$ "nontraced" qubits belonging to Bob, has the same form as Eq. (12). Therefore, repeating the above single-qubit tracing procedure, one finds that the density operator for any qubit $i^{\prime \prime}$ belonging to Bob $\left(i^{\prime \prime}=1^{\prime \prime}, 2^{\prime \prime}, \ldots\right.$, or $\left.m^{\prime \prime}\right)$ can, after tracing over Bob's other $m-1$ qubits, be written as

$$
\rho_{i^{\prime \prime}}=\left|\alpha_{i}\right|^{2}|0\rangle\left\langle\left. 0|+| \beta_{i}\right|^{2} \mid 1\right\rangle\langle 1|
$$

in the case when Alice measures the qubit pair $\left(i, i^{\prime}\right)$ in either Bell state $\left|\phi_{i i^{\prime}}^{+}\right\rangle$or $\left|\phi_{i i^{\prime}}^{-}\right\rangle$. On the other hand,

$$
\rho_{i^{\prime \prime}}=\left|\alpha_{i}\right|^{2}|1\rangle\left\langle\left. 1|+| \beta_{i}\right|^{2} \mid 0\right\rangle\langle 0|
$$

in the case when the pair $\left(i, i^{\prime}\right)$ is measured in the Bell state $\left|\psi_{i i^{\prime}}^{+}\right\rangle$or $\left|\psi_{i i^{-}}\right\rangle$. The above process demonstrates that the density operator (15) and (16) depends only on the outcome of Alice's Bell-state measurement on the pair $\left(i, i^{\prime}\right)$, but independent of the outcome of Alice's Bell-state measurement on all other pairs.

Equations (15) and (16) demonstrate the following results. First, any qubit $i^{\prime \prime}$ belonging to Bob is in a mixed state, in which it is in the state $|0\rangle$ with a probability $\left|\alpha_{i}\right|^{2}$ or $\left|\beta_{i}\right|^{2}$ (depending on Alice's Bell-state measurement outcome), while being in the state $|1\rangle$ with a probability $\left|\beta_{i}\right|^{2}$ or $\left|\alpha_{i}\right|^{2}$. Second, Bob has amplitude information about Alice's each message qubit, but knows nothing about its phase. Therefore, in general (i.e., $\alpha_{i} \neq 0$ or $\beta_{i} \neq 0$ for $i=1,2, \ldots, m$ ), Bob cannot fully restore the original state of each message qubit belonging to Alice, even if one agent does not cooperate with him.

It is necessary for us to compare the present method with the method in $[13,14]$. From the description above, we conclude that to teleport $m$-qubit information to a distant receiver via the control of one agent, the present method requires only the following:

(i) $2(m+1)$ auxiliary qubits for the preparation of the state (2),

(ii) one qubit being assigned to the controller,

(iii) one single-qubit Hadamard transformation and one single-qubit measurement being performed by the controller,

(iv) one-bit classical message being sent to the receiver. However, as mentioned in the Introduction, to implement the present task, the method in $[13,14]$ will require the following:

(i) $3 m$ auxiliary qubits for preparing $m$ copies of a threequbit GHZ state,

(ii) $m$ qubits being assigned to the controller, (iii) $m$ single-qubit Hadamard transformations and $m$ single-qubit measurements being performed by the controller,

(iv) $m$-bit classical messages being sent to the receiver by the controller.

The above analysis demonstrates that for the case of $m$ $=1$, the present protocol is trivial, since it requires four auxiliary qubits while the method in $[13,14]$ only needs three auxiliary qubits. However, the advantage for the present proposal appears when $m=2$, because it requires the same number of auxiliary qubits but less local operation and classical communication, compared with the method in $[13,14]$. Moreover, when $m=3$, the number of auxiliary qubits required in the present method becomes smaller than that using the method in $[13,14]$. One can clearly see that the advantage of the present method becomes apparent with increments of $m$. Especially, when $m$ is a large number, the required auxiliary qubit resources, local operations by the controller, and classical communication between the controller and the receiver are greatly reduced in the present approach.

On a final note, we point out that the number of Alice's Bell-state measurements needed in the present protocol is the same as that required by the method in $[13,14]$. This is obvious, since using the method in $[13,14]$, Alice also needs to perform a series of Bell-state measurements, each acting on one message qubit and one GHZ qubit.

\section{TELEPORTATION OF MULTIQUBIT INFORMATION TO A DISTANT RECEIVER VIA THE CONTROL OF MANY AGENTS}

In this section, we discuss how to decompose multiqubit GHZ states and how to generalize the above method to the teleportation of multiqubit information via the control of many agents.

\section{A. Decomposition of multiqubit GHZ states}

In the past few years, GHZ states have been extensively studied by many researchers. They play an important role in quantum information processing and communication. Many theoretical proposals have appeared for the generation of multiqubit GHZ states. Moreover, it has been reported that up to four-qubit GHZ states were experimentally prepared with polarized-state photons [23] and trapped ions [24]. As especially relevant to this work, we consider the following two types of $(n+1)$-qubit GHZ states:

$$
\begin{aligned}
|\mathrm{GHZ}\rangle_{+} & =|00 \cdots 0\rangle+|11 \cdots 1\rangle, \\
|\mathrm{GHZ}\rangle_{-} & =|00 \cdots 0\rangle-|11 \cdots 1\rangle .
\end{aligned}
$$

We find that if a Hadamard transformation is performed on each qubit, the states (17) and (18) will be decomposed, respectively, into

$$
|\mathrm{GHZ}\rangle_{+} \rightarrow \sum_{\left\{x_{l}\right\}}\left|\left\{x_{l}\right\}\right\rangle|0\rangle+\sum_{\left\{y_{l}\right\}}\left|\left\{y_{l}\right\}\right\rangle|1\rangle,
$$




$$
|\mathrm{GHZ}\rangle_{-} \rightarrow \sum_{\left\{x_{l}\right\}}\left|\left\{x_{l}\right\}\right\rangle|1\rangle+\sum_{\left\{y_{l}\right\}}\left|\left\{y_{l}\right\}\right\rangle|0\rangle,
$$

where $\left|\left\{x_{l}\right\}\right\rangle=\left|x_{1} x_{2} \cdots x_{n}\right\rangle$ and $\left|\left\{y_{l}\right\}\right\rangle=\left|y_{1} y_{2} \cdots y_{n}\right\rangle$ are computational basis states of the first $n$ qubits $\left(x_{l}, y_{l} \in\{0,1\}, l\right.$ $=1,2, \ldots, n)$, and $\left.\left.\Sigma_{\left\{x_{l}\right\}}\right\}\left\{x_{l}\right\}\right\rangle\left(\Sigma_{\left\{y_{l}\right\}}\left|\left\{y_{l}\right\}\right\rangle\right)$ is a sum over all possible basis states $\left|\left\{x_{l}\right\}\right\rangle\left(\left|\left\{y_{l}\right\}\right\rangle\right)$ each containing an even (odd) number of "1"s. For instance, when $n=4, \quad \sum_{\left\{x_{l}\right\}}\left|\left\{x_{l}\right\}\right\rangle$ $=|0000\rangle+|1100\rangle+|1010\rangle+\cdots+|1111\rangle$. Note that the number of the basis states $\left|\left\{x_{l}\right\}\right\rangle$ is the same as that of the basis states $\left|\left\{y_{l}\right\}\right\rangle$; thus, the states (19) and (20) on the right side both have the same normalized factors.

\section{B. Teleportation of $m$-qubit information via the control of $n$ agents}

Now, suppose that Alice has a string of message qubits labeled by $1,2, \ldots, m$, which is initially in the state $\prod_{i=1}^{m}\left(\alpha_{i}|0\rangle_{i}+\beta_{i}|1\rangle_{i}\right)$. She wishes to send $m$-qubit information to Bob via the control of $n$ agents $\left(A_{1}, A_{2}, \ldots, A_{n}\right)$ in a network, such that Bob can get the complete information of each message qubit only if all the agents collaborate. This can be done by the following procedure:

First, Alice prepares the following EPR-GHZ entangled state through local logic gates:

$$
\begin{aligned}
& \prod_{i=1}^{m}\left(|00\rangle_{i^{\prime} i^{\prime \prime}}+|11\rangle_{i^{\prime} i^{\prime \prime}}\right) \otimes|\mathrm{GHZ}\rangle_{+}+\prod_{i=1}^{m}\left(|00\rangle_{i^{\prime} i^{\prime \prime}}-|11\rangle_{i^{\prime} i^{\prime \prime}}\right) \\
& \otimes|\mathrm{GHZ}\rangle_{-},
\end{aligned}
$$

[where $|\mathrm{GHZ}\rangle_{ \pm}=|00 \cdots 0\rangle_{ \pm}|11 \cdots 1\rangle$ are $(n+1)$-qubit $\mathrm{GHZ}$ states], and then she sends the first $n$ GHZ qubits to the $n$ agents and the $m$ EPR qubits $\left(1^{\prime \prime}, 2^{\prime \prime}, \ldots, m^{\prime \prime}\right)$ to Bob, while keeping the last GHZ qubit and the other $m$ EPR qubits $\left(1^{\prime}, 2^{\prime}, \ldots, m^{\prime}\right)$ to herself. The state of the whole system is given by

$$
\begin{aligned}
& \prod_{i=1}^{m}\left[\left(\alpha_{i}|0\rangle_{i}+\beta_{i}|1\rangle_{i}\right)\left(|00\rangle_{i^{\prime} i^{\prime \prime}}+|11\rangle_{i^{\prime} i^{\prime \prime}}\right)\right] \otimes|\mathrm{GHZ}\rangle_{+} \\
& +\prod_{i=1}^{m}\left[\left(\alpha_{i}|0\rangle_{i}+\beta_{i}|1\rangle_{i}\right)\left(|00\rangle_{i^{\prime} i^{\prime \prime}}-|11\rangle_{i^{\prime} i^{\prime \prime}}\right)\right] \otimes|\mathrm{GHZ}\rangle_{-} .
\end{aligned}
$$

Second, Alice performs a series of two-qubit Bell-state measurements, respectively, on $m$ qubit pairs $\left(1,1^{\prime}\right),\left(2,2^{\prime}\right), \ldots,\left(m, m^{\prime}\right)$. After that, we have

$$
|\psi\rangle|\mathrm{GHZ}\rangle_{+}+\left|\psi^{\prime}\right\rangle|\mathrm{GHZ}\rangle_{-},
$$

where $|\psi\rangle$ and $\left|\psi^{\prime}\right\rangle$ are the states for the $m$ qubits $\left(1^{\prime \prime}, 2^{\prime \prime}, \ldots, m^{\prime \prime}\right)$ belonging to Bob. Note that the left part of the first (second) product term in Eq. (22) is the same as that of the first (second) product term in Eq. (3). Thus, the two states $|\psi\rangle$ and $\left|\psi^{\prime}\right\rangle$ here take the same form as $|\psi\rangle$ and $\left|\psi^{\prime}\right\rangle$ described by Eq. (7), respectively.

Third, each agent and Alice perform a Hadamard transformation on their respective GHZ qubits. After that, based on Eqs. (19) and (20), one gets, from Eq. (23),

$$
\begin{aligned}
& |\psi\rangle\left[\sum_{\left\{x_{l}\right\}}\left|\left\{x_{l}\right\}\right\rangle|0\rangle+\sum_{\left\{x_{l}\right\}}\left|\left\{y_{l}\right\}\right\rangle|1\rangle\right]+\left|\psi^{\prime}\right\rangle\left[\sum_{\left\{x_{l}\right\}}\left|\left\{x_{l}\right\}\right\rangle|1\rangle\right. \\
& \left.+\sum_{\left\{y_{l}\right\}}\left|\left\{y_{l}\right\}\right\rangle|0\rangle\right] .
\end{aligned}
$$

Last, each agent and Alice make a measurement on their respective GHZ qubits and then send their measurement results to Bob. Recall the notation of $\left|\left\{x_{l}\right\}\right\rangle$ and $\left|\left\{y_{l}\right\}\right\rangle$ described above; i.e., each basis state $\left|\left\{x_{l}\right\}\right\rangle\left(\left|\left\{y_{l}\right\}\right\rangle\right)$ contains an even $(o d d)$ number of "1"'s. Therefore, one sees from Eq. (24) that Bob can predict that the $m$ qubits $\left(1^{\prime \prime}, 2^{\prime \prime}, \ldots, m^{\prime \prime}\right)$ belonging to him must be in the state $|\psi\rangle\left(\left|\psi^{\prime}\right\rangle\right)$, if he knows that the outcome of the $n$ agents'measurement on their $n$ GHZ qubits contains an even number of " 1 "'s and that Alice measured her GHZ qubit in the state $|0\rangle(|1\rangle)$. On the other hand, the result (24) shows that Bob knows that his $m$ qubits $\left(1^{\prime \prime}, 2^{\prime \prime}, \ldots, m^{\prime \prime}\right)$ must be in the state $|\psi\rangle\left(\left|\psi^{\prime}\right\rangle\right)$, if he knows that the outcome of the $n$ agents' measurement includes an odd number of " 1 ""s and that Alice measured her GHZ qubit in the state $|1\rangle(|0\rangle)$. Hence, according to the measurement outcomes from the $n$ agents and Alice, Bob can predict whether his $m$ qubits are in $|\psi\rangle$ or $\left|\psi^{\prime}\right\rangle$. As addressed in the previous section, Bob can restore the original state of $m$ message qubits $(1,2, \ldots, m)$ from the state $|\psi\rangle$ or $\left|\psi^{\prime}\right\rangle$ of the $m$ qubits $\left(1^{\prime \prime}, 2^{\prime \prime}, \ldots, m^{\prime \prime}\right)$, according to the Bell-state measurement outcome from Alice and through his local single-qubit logic operations.

We have shown, based on (23), that the quantum message originally carried by the $m$ message qubits $(1,2, \ldots, m)$ can be recovered by Bob, as long as each agent performs a Hadamard transformation and then a measurement on his/her qubit. Now let us focus on the problem that Bob can not gain the full quantum message even if one agent does not collaborate. To see this, let us go back to the state (23). This state can be rewritten as

$|\psi\rangle\left[\left(\left|\phi^{+}\right\rangle+\left|\phi^{-}\right\rangle\right)|0\rangle_{A_{i}}+\left(\left|\phi^{+}\right\rangle-\left|\phi^{-}\right\rangle\right)|1\rangle_{A_{i}}\right]+\left|\psi^{\prime}\right\rangle\left[\left(\left|\phi^{+}\right\rangle+\left|\phi^{-}\right\rangle\right)\right.$

$\left.\times|0\rangle_{A_{i}}-\left(\left|\phi^{+}\right\rangle-\left|\phi^{-}\right\rangle\right)|1\rangle_{A_{i}}\right]$,

where $|0\rangle_{A_{i}}$ and $|1\rangle_{A_{i}}$ are the two logic states of the GHZ qubit belonging to agent $A_{i}(i=1,2, \ldots$, or $n)$, while $\left|\phi^{+}\right\rangle$and $\left|\phi^{-}\right\rangle$, taking the form of Eqs. (17) and (18), respectively, are the GHZ states of the remaining $n \mathrm{GHZ}$ qubits belonging to other $n-1$ agents and Alice.

Assume the agent $A_{i}$ does not collaborate with Bob. When the other $n-1$ agents and Alice perform a Hadamard transformation on their respective GHZ qubits, it follows from Eqs. (19) and (20) that the states $\left|\phi^{+}\right\rangle$and $\left|\phi^{-}\right\rangle$will be transformed into

$$
\begin{gathered}
\left|\phi^{+}\right\rangle \rightarrow \sum_{\left\{x_{l}^{\prime}\right\}}\left|\left\{x_{l}^{\prime}\right\}\right\rangle|0\rangle_{a}+\sum_{\left\{y_{l}^{\prime}\right\}}\left|\left\{y_{l}^{\prime}\right\}\right\rangle|1\rangle_{a}, \\
\left|\phi^{-}\right\rangle \rightarrow \sum_{\left\{x_{l}^{\prime}\right\}}\left|\left\{x_{l}^{\prime}\right\}\right\rangle|1\rangle_{a}+\sum_{\left\{y_{l}^{\prime}\right\}}\left|\left\{y_{l}^{\prime}\right\}\right\rangle|0\rangle_{a},
\end{gathered}
$$

where the subscript $a$ represents the GHZ qubit belonging to Alice; $\left|\left\{x_{l}^{\prime}\right\}\right\rangle=\left|x_{1}^{\prime} x_{2}^{\prime} \ldots x_{n-1}^{\prime}\right\rangle$ and $\left|\left\{y_{l}^{\prime}\right\}\right\rangle=\left|y_{1}^{\prime} y_{2}^{\prime} \ldots y_{n-1}^{\prime}\right\rangle$ are com- 
putational basis states of the $n-1 \mathrm{GHZ}$ qubits belonging to the other $n-1$ agents $\left(x_{l}^{\prime}, y_{l}^{\prime} \in\{0,1\} ; l=1,2, \ldots n-1\right)$. Further, $\Sigma_{\left\{x_{l}^{\prime}\right\}}\left\{\left\{x_{l}^{\prime}\right\}\right\rangle\left(\Sigma_{\left\{y_{l}^{\prime}\right\}}\left|\left\{y_{l}^{\prime}\right\}\right\rangle\right)$ represents a sum over all possible basis states $\left|\left\{x_{l}^{\prime}\right\}\right\rangle\left(\left|\left\{y_{l}^{\prime}\right\}\right\rangle\right)$ each containing an even (odd) number of "1"'s. The state (25) will, after replacing $\left|\phi^{ \pm}\right\rangle$by Eq. (26), change into

$$
\begin{aligned}
& {\left[\left(|\psi\rangle+\left|\psi^{\prime}\right\rangle\right)|0\rangle_{A_{i}}+\left(|\psi\rangle-\left|\psi^{\prime}\right\rangle\right)|1\rangle_{A_{i}}\right] \sum_{\left\{x_{l}^{\prime}\right\}}\left|\left\{x_{l}^{\prime}\right\}\right\rangle|0\rangle_{a}+[(|\psi\rangle} \\
& \left.\left.+\left|\psi^{\prime}\right\rangle\right)|0\rangle_{A_{i}}-\left(|\psi\rangle-\left|\psi^{\prime}\right\rangle\right)|1\rangle_{A_{i}}\right] \sum_{\left\{x_{l}^{\prime}\right\}}\left|\left\{x_{l}^{\prime}\right\}\right\rangle|1\rangle_{a}+\left[\left(|\psi\rangle+\left|\psi^{\prime}\right\rangle\right)\right. \\
& \left.\times|0\rangle_{A_{i}}-\left(|\psi\rangle-\left|\psi^{\prime}\right\rangle\right)|1\rangle_{A_{i}}\right] \sum_{\left\{y_{l}^{\prime}\right\}}\left|\left\{y_{l}^{\prime}\right\}\right\rangle|0\rangle_{a}+\left[\left(|\psi\rangle+\left|\psi^{\prime}\right\rangle\right)|0\rangle_{A_{i}}\right. \\
& \left.+\left(|\psi\rangle-\left|\psi^{\prime}\right\rangle\right)|1\rangle_{A_{i}}\right] \sum_{\left\{y_{l}^{\prime}\right\}}\left|\left\{y_{l}^{\prime}\right\}\right\rangle|1\rangle_{a},
\end{aligned}
$$

which implies that if the other $n-1$ agents and Alice perform a measurement on their respective GHZ qubits, the $m$ qubits $\left(1^{\prime \prime}, 2^{\prime \prime}, \ldots, m^{\prime \prime}\right)$ belonging to Bob will be entangled with agent $A_{i}$ 's GHZ qubit.

From Eq. (27), it is easily seen that for every outcome $\left|\left\{x_{l}^{\prime}\right\}\right\rangle|0\rangle_{a},\left|\left\{x_{l}^{\prime}\right\}\right\rangle|1\rangle_{a},\left|\left\{y_{l}^{\prime}\right\}\right\rangle|0\rangle_{a}$, or $\left|\left\{y_{l}^{\prime}\right\}\right\rangle|1\rangle_{a}$ of the other $n-1$ agents' and Alice's measurements on their GHZ qubits, the density operator of the $m$ qubits $\left(1^{\prime \prime}, 2^{\prime \prime}, \ldots, m^{\prime \prime}\right)$ belonging to Bob is, after tracing over agent $A_{i}$ 's GHZ qubit, given by

$$
\rho=\left(|\psi\rangle+\left|\psi^{\prime}\right\rangle\right)\left(\left\langle\psi\left|+\left\langle\psi^{\prime}\right|\right)+\left(|\psi\rangle-\left|\psi^{\prime}\right\rangle\right)\left(\langle\psi|-\left\langle\psi^{\prime}\right|\right) .\right.\right.
$$

Since Eq. (28) takes the same form as Eq. (12), one can obtain the same results (13)-(16) as described above. Therefore, Bob can fully restore the original state of $m$ message qubits $(1,2, \ldots, m)$, only if all the agents collaborate with him.

In summary, to teleport $m$-qubit information to a distant receiver via the control of $n$ agents, the present method requires only the following:

(i) $2 m+n+1$ auxiliary qubits for preparing the state (21);

(ii) one qubit being distributed to each agent;

(iii) one single-qubit Hadamard transformation and one single-qubit measurement being performed by each agent;

(iv) one-bit classical message being sent to the receiver by each agent.

In contrast, to implement the same task, the method in $[13,14]$ requires the following:

(i) $m(n+2)$ auxiliary qubits for preparing $m$ copies of a $(n+2)$-qubit GHZ state;

(ii) $m$ qubits being distributed to each agent;

(iii) $m$ single-qubit Hadamard transformations and $m$ single-qubit measurements being performed by each agent;

(iv) $m$-bit classical message being sent to the receiver by each agent.

For the case of $m=1$, the present method is not interesting since it requires one more auxiliary qubit than the method in $[13,14]$. However, the advantage of the present proposal appears when $m=2$ and becomes apparent as $m$ increases.

\section{CONTROLLED TELEPORTATION OF MULTIPLE QUBIT-STRING INFORMATION TO MANY DISTANT RECEIVERS}

It is interesting to note that the method described above can be further extended to teleport multiple qubit-string information to many distant receivers via the control of many agents in a network. Suppose that Alice holds $k$ qubit strings labeled by $1,2, \ldots, k$. The qubit string $l$ contains $m_{l}$ message qubits, which is initially in the state $\prod_{i=1}^{m_{l}}\left(\alpha_{i, l}|0\rangle_{i, l}+\beta_{i, l}|1\rangle_{i, l}\right)$ $(l=1,2, \ldots, k)$. The state of the $k$ qubit strings is given by $\Pi_{l=1}^{k} \prod_{i=1}^{m_{l}}\left(\alpha_{i, l}|0\rangle_{i, l}+\beta_{i, l}|1\rangle_{i, l}\right)$. Now, Alice wishes to teleport the $k$ qubit-string information to $k$ distant receivers (the message carried by the qubit string $l$ is for the receiver $l$ ) via the control of $n$ agents in a network, such that each receiver can fully recover the original quantum message of the corresponding qubit string only if all the agents cooperate. The present task can be implemented with the following EPRGHZ entangled state:

$$
\begin{aligned}
& \prod_{l=1}^{k} \prod_{i=1}^{m_{l}}\left(|00\rangle_{i^{\prime} i^{\prime \prime}, l}+|11\rangle_{i^{\prime} i^{\prime \prime}, l}\right) \otimes|\mathrm{GHZ}\rangle_{+}+\prod_{l=1}^{k} \prod_{i=1}^{m_{l}}\left(|00\rangle_{i^{\prime} i^{\prime \prime}, l}\right. \\
& \left.-|11\rangle_{i^{\prime} i^{\prime \prime}, l}\right) \otimes|\mathrm{GHZ}\rangle_{-},
\end{aligned}
$$

where $|\mathrm{GHZ}\rangle_{ \pm}$are the GHZ states (17) and (18) of the (n +1) GHZ qubits shared by the $n$ agents and Alice; the $m_{l}$ EPR qubits $\left(1^{\prime \prime}, 2^{\prime \prime}, \ldots, m_{l}^{\prime \prime}\right)$ for the set $l$ belong to the receiver $l$, while the other $m_{l}$ EPR qubits $\left(1^{\prime}, 2^{\prime}, \ldots, m_{l}^{\prime}\right)$ for the set $l$ are kept by Alice. Here, the set $l$ represents the "qubit string $l$ and the $m_{l}$ EPR pairs shared by Alice and the receiver $l "(l=1,2, \ldots, k)$. The state of the whole system is thus given by

$$
\begin{aligned}
& \prod_{l=1}^{k} \prod_{i=1}^{m_{l}}\left[\left(\alpha_{i, l}|0\rangle_{i, l}+\beta_{i, l}|1\rangle_{i, l}\right)\left(|00\rangle_{i^{\prime} i^{\prime \prime}, l}+|11\rangle_{i^{\prime} i^{\prime \prime}, l}\right)\right] \otimes|\mathrm{GHZ}\rangle_{+} \\
& +\prod_{l=1}^{k} \prod_{i=1}^{m_{l}}\left[\left(\alpha_{i, l}|0\rangle_{i, l}+\beta_{i, l}|1\rangle_{i, l}\right)\left(|00\rangle_{i^{\prime} i^{\prime \prime}, l}-|11\rangle_{i^{\prime} i^{\prime \prime}, l}\right)\right] \otimes|\mathrm{GHZ}\rangle_{-} .
\end{aligned}
$$

Now, Alice performs a series of two-qubit Bell-state measurements, which are, respectively, on qubit pairs $\left(1,1^{\prime}\right),\left(2,2^{\prime}\right), \ldots,\left(m_{l}, m_{l}^{\prime}\right)$ for the set $l$. After that, we obtain

$$
\prod_{l=1}^{k}|\psi\rangle_{l} \otimes|\mathrm{GHZ}\rangle_{+}+\prod_{l=1}^{k}\left|\psi^{\prime}\right\rangle_{l} \otimes|\mathrm{GHZ}\rangle_{-}
$$

where $|\psi\rangle_{l}=\prod_{i=1}^{m_{l}}|\psi\rangle_{i^{\prime \prime}, l}$ and $\left|\psi^{\prime}\right\rangle_{l}=\prod_{i=1}^{m}\left|\psi^{\prime}\right\rangle_{i^{\prime \prime}, l}$ are the states for the $m_{l}$ qubits $\left(1^{\prime \prime}, 2^{\prime \prime}, \ldots, m_{l}^{\prime \prime}\right)$ belonging to the receiver $l$. Here $|\psi\rangle_{i^{\prime \prime}, l}$ and $\left|\psi^{\prime}\right\rangle_{i^{\prime \prime}, l}$ are the states of qubit $i^{\prime \prime}$ for the receiver $l$, which depend on the outcome of Alice's Bell-state measurement on the associated qubit pair $\left(i, i^{\prime}\right)$ for the set $l$, and take the form of Eqs. (8) and (9), respectively.

Note that the states $|\psi\rangle_{l}$ and $\left|\psi^{\prime}\right\rangle_{l}$ have the same form as $|\psi\rangle$ and $\left|\psi^{\prime}\right\rangle$ described in Eqs. (7), respectively. Therefore, based on Eq. (31) and using the above procedure, it is straightforward to show that quantum information originally 
carried by each qubit string can be recovered by the corresponding receiver, with the aid of all the agents.

In order for each receiver to restore the original state of the corresponding qubit string, the following procedure can be followed: (i) Each agent and Alice need to perform a Hadamard transformation and then a measurement on their respective GHZ qubits. (ii) Each agent and Alice need to send each receiver their measurement results on their GHZ qubits. And (iii) Alice needs to send the receiver $l$ the outcome of her Bell-state measurements on the qubit pairs $\left(1,1^{\prime}\right),\left(2,2^{\prime}\right), \ldots,\left(m_{l}, m_{l}^{\prime}\right)$ for the set $l$, so that the receiver $l$ can recover the original state of the qubit string $l$.

On the other hand, it can be shown from Eq. (31) that even if one agent does not collaborate, the density operator for each qubit belonging to each receiver takes the form of Eq. (15) or (16); i.e., no receiver can fully restore the original state of the corresponding "message qubit string" without the cooperation of all the agents.

To realize the present task, the present method requires only the following:

(i) $2 \sum_{l=1}^{k} m_{l}+n+1$ auxiliary qubits for preparing the state

(ii) one qubit being assigned to each agent;

(iii) one single-qubit Hadamard transformation and one single-qubit measurement being performed by each agent;

(iv) one-bit classical message being sent to each receiver by each agent.

In contrast, to implement the same task, the method in $[13,14]$ requires the following:

(i) $\sum_{l=1}^{k} m_{l}(n+2)$ auxiliary qubits for preparing $\sum_{l=1}^{k} m_{l}$ copies of a $(n+2)$-qubit GHZ state;

(ii) $\sum_{l=1}^{k} m_{l}$ qubits being assigned to each agent;

(iii) $\sum_{l=1}^{k} m_{l}$ single-qubit Hadamard transformations and $\sum_{l=1}^{k} m_{l}$ single-qubit measurements being performed by each agent;

(iv) $m_{l}$-bit classical message being sent to the receiver $l$ by each agent.

One can see that even for $m_{l}=1$ and $k=2$, the present method is effective, since (a) the number of qubits distributed to each agent, the number of Hadamard transformation by each agent, or the number of measurement by each agent is 1, which is, however, 2 for the method in [13,14], and (b) the number of auxiliary qubits required is $n+5$, which is smaller than $2 n+4$ needed in the method in $[13,14]$, when $n>1$. More interestingly, with the increment of $m_{l}, k$, or $n$, the advantage of the present method becomes very apparent.

\section{DISCUSSION AND CONCLUSION}

It should be pointed out that as far as the control efficiency of each agent on teleportation, the present scheme is identical to those in $[13,14]$. This is because the results (15) and (16) applied in the present proposal are the same as those employed in $[13,14]$. However, as shown above, the present scheme is extremely simple and economical in the realization of multiqubit quantum information teleportation via the control of many agents in a network.

The scheme presented here works essentially through having originally nonentangled quantum information, carried by any two message qubits, to be entangled each other after Alice performs a series of Bell-state measurements. This can be seen from Eq. (23). For example, let us consider a $m=2$ case-i.e., teleporting the state $\left(\alpha_{1}|0\rangle_{1}+\beta_{1}|1\rangle_{1}\right) \otimes\left(\alpha_{2}|0\rangle_{2}\right.$ $\left.+\beta_{2}|1\rangle_{2}\right)$ of the two message qubits $(1,2)$ to Bob. Based on Eqs. (7)-(9), one sees that if Alice measures the two qubits $\left(1,1^{\prime}\right)$ and another two qubits $\left(2,2^{\prime}\right)$ in the Bell states-e.g., $\left|\phi_{11^{\prime}}^{+}\right\rangle$and $\left|\phi_{22^{\prime}}^{+}\right\rangle$—the state (23) for the remaining qubit system will be

$$
\begin{gathered}
\left(\alpha_{1}|0\rangle_{1^{\prime \prime}}+\beta_{1}|1\rangle_{1^{\prime \prime}}\right)\left(\alpha_{2}|0\rangle_{2^{\prime \prime}}+\beta_{2}|1\rangle_{2^{\prime \prime}}\right) \otimes|\mathrm{GHZ}\rangle_{+}+\left(\alpha_{1}|0\rangle_{1^{\prime \prime}}\right. \\
\left.-\beta|1\rangle_{1^{\prime \prime}}\right)\left(\alpha_{2}|0\rangle_{2^{\prime \prime}}-\beta_{2}|1\rangle_{2^{\prime \prime}}\right) \otimes|\mathrm{GHZ}\rangle_{-} .
\end{gathered}
$$

The result (32) implies that if Bob measures the qubit $1^{\prime \prime}$ in the state $\alpha_{1}|0\rangle+\beta_{1}|1\rangle$, he can predict that his qubit $2^{\prime \prime}$ must be in the state $\alpha_{2}|0\rangle+\beta_{2}|1\rangle$. On the other hand, if Bob detects the qubit $1^{\prime \prime}$ in the state $\alpha_{1}|0\rangle-\beta_{1}|1\rangle$, he knows that his qubit $2^{\prime \prime}$ must be in the state $\alpha_{2}|0\rangle-\beta_{2}|1\rangle$. Therefore, after Alice performs Bell-state measurements, the quantum information originally carried by the two message qubits $(1,2)$ is not only transferred onto Bob's qubits $\left(1^{\prime \prime}, 2^{\prime \prime}\right)$ but also becomes entangled with each other.

In Refs. [13,14], single-qubit measurements are carried out in a basis $|+\rangle=|0\rangle+|1\rangle$ and $|-\rangle=|0\rangle-|1\rangle$. In contrast, as shown above, the present single-qubit measurement is performed in the basis $|0\rangle$ and $|1\rangle$. For certain kinds of qubits (e.g., superconducting charge or flux qubits), it is rather hard to make a measurement in the basis $|+\rangle$ and $|-\rangle$, but straightforward in the basis $|0\rangle$ and $|1\rangle$. As a matter of fact, based on $|0\rangle=|+\rangle+|-\rangle$ and $|1\rangle=|+\rangle-|-\rangle$, it is noted that Hadamard transformations are not necessary in the present proposal, because the same results can be obtained when each agent performs a measurement on his or her qubit in the basis $|+\rangle$ and $|-\rangle$, instead of a Hadamard transformation followed by a measurement in the basis $|0\rangle$ and $|1\rangle$, and then sends his or her measurement result $|+\rangle$ or $|-\rangle$ (one-bit classical message) to the receiver(s).

Another point may need to be made here. As shown above, Alice's Bell-state measurement, Alice's single-qubit operation (Hadamard transformation/measurement), and each agent's operation are independently performed on different qubits. Therefore, like the method in $[13,14]$, the present proposal actually does not require the operating order among Alice's Bell-state measurement, Alice's single-qubit operation, and each agent's operation.

Although we shall not attempt here a comprehensive study of the security of the scheme against all possible forms of eavesdropping and/or cheating, we believe that it is probably quite secure, for several reasons. First, the eavesdropping by entangling ancillary qubits with Bob's qubits can be revealed by comparing a subset of the states Bob received to the ones Alice sent. Second, the qubits Alice sends to Bob are basically useless without the classical information possessed by Alice. Hence, even if Eve were to intercept the qubits intended for Bob and replace them by fakes and somehow eavesdropped on the (classical) communication channels through which all the agents disclose to Bob their measurement results, she would still not be able to recover the message qubits' original states without access to Alice's clas- 
sical information (her measurement outcomes), given that Alice sends her classical information to Bob using standard quantum cryptography [14]. It is conceivable that an eavesdropper might obtain partial information by entangling enough ancillary qubits with the qubits belonging to all the agents and Bob, but presumably such entanglement could be detected by tests conducted on "sample" EPR-GHZ entangled states initially shared by Alice and the other parties.

In summary, we have presented a new method for teleporting multiqubit quantum information from a sender to a distant receiver via the control of many agents in a network. A special feature of our entangling quantum information concept is that to implement a control of multiqubit quantum information teleportation, the present scheme needs to assign only one qubit to each agent, followed by every agent performing only one Hadamard transformation and one measurement, and then sending only one-bit classical message to the receiver. As a result, the required auxiliary qubit resources, the number of local operations, and the quantity of classical communication are greatly reduced in the present proposal. The method presented here can also be extended to implement a multiparty controlled teleportation of multiple qubit-string quantum information to many distant receivers. We believe that our scheme is of considerable interest, especially because of its relatively straightforward nature in realizing simultaneous control of multiqubit quantum information teleportation in an efficient and simple manner.

\section{ACKNOWLEDGMENTS}

C.P.Y. is very grateful to Julio Gea-Banacloche for useful discussions and Phyllis Bia for the help in the preparation of this manuscript. This research was partially supported by National Science Foundation QuBIC program (Grant No. ECS0201995), ITR program (Grant No. DMR-0325551), and AFOSR (Grant No. F49620-01-1-0439), funded under the Department of Defense University Research Initiative on Nanotechnology (DURINT) Program and by the ARDA.
[1] C. H. Bennett, G. Brassard, C. Crépeau, R. Jozsa, A. Peres, and W. K. Wootters, Phys. Rev. Lett. 70, 1895 (1993).

[2] M. Fujii, Phys. Rev. A 68, 050302 (2003).

[3] N. Ba An, Phys. Rev. A 68, 022321 (2003).

[4] W. P. Bowen, N. Treps, B. C. Buchler, R. Schnabel, T. C. Ralph, Hans-A. Bachor, T. Symul, and P. K. Lam, Phys. Rev. A 67, 032302 (2003).

[5] T. J. Johnson, S. D. Bartlett, and B. C. Sanders, Phys. Rev. A 66, 042326 (2002).

[6] J. Fang, Y. Lin, S. Zhu, and X. Chen, Phys. Rev. A 67, 014305 (2003).

[7] W. Son, J. Lee, M. S. Kim, and Y.-J. Park, Phys. Rev. A 64, 064304 (2001).

[8] E. F. Galvão and L. Hardy, Phys. Rev. A 62, 012309 (2000).

[9] C. P. Yang and G. C. Guo, Chin. Phys. Lett. 16, 628 (1999); 17, 162 (2000).

[10] D. Bouwmeester, J. W. Pan, K. Mattle, M. Eibl, H. Weinfurter, and A. Zeilinger, Nature (London) 390, 575 (1997).

[11] A. Furusawa, J. L. Sørensen, S. L. Braunstein, C. A. Fuchs, H. J. Kimble, and E. S. Polzik, Science 282, 706 (1998).

[12] M. A. Nielsen, E. Knill, and R. Laflamme, Nature (London) 396, 52 (1998).

[13] A. Karlsson and M. Bourennane, Phys. Rev. A 58, 4394
(1998).

[14] M. Hillery, V. Buzek, and A. Berthiaume, Phys. Rev. A 59, 1829 (1999).

[15] R. Cleve, D. Gottesman, and H. K. Lo, Phys. Rev. Lett. 83, 648 (1999).

[16] A. Karlsson, M. Koashi, and N. Imoto, Phys. Rev. A 59, 162 (1999).

[17] S. Bandyopadhyay, Phys. Rev. A 62, 012308 (2000).

[18] D. Gottesman, Phys. Rev. A 61, 042311 (2000).

[19] Li-Yi Hsu, Phys. Rev. A 68, 022306 (2003).

[20] A. C. A. Nascimento, J. M. Quade, and H. Imai, Phys. Rev. A 64, 042311 (2001).

[21] B. Aoun and M. Tarifi, e-print quant-ph/0401076.

[22] E. Biham, B. Huttner, and T. Mor, Phys. Rev. A 54, 2651 (1996);P. D. Townsend, Nature (London) 385, 47 (1997);S. Bose, V. Vedral, and P. L. Knight, Phys. Rev. A 57, 822 (1998).

[23] J. W. Pan, M. Daniell, S. Gasparoni, G. Weihs, and A. Zeilinger, Phys. Rev. Lett. 86, 4435 (2001).

[24] C. A. Sackett, D. Kielpinski, B. E. King, C. Langer, V. Meyer, C. J. Myatt, M. Rowe, Q. A. Turchette, W. M. Itano, D. J. Wineland, and C. Monroe, Nature (London) 404, 256 (2000). 